\title{
IP Lookups Using Multiway and Multicolumn Search
}

\author{
Butler Lampson, Venkatachary Srinivasan, and George Varghese, Associate Member, IEEE
}

\begin{abstract}
IP address lookup is becoming critical because of increasing routing table sizes, speed, and traffic in the Internet. Given a set $S$ of prefixes and an IP address $D$, the $I P$ address lookup problem is to find the longest matching prefix of $D$ in set $S$. This paper shows how binary search can be adapted for solving the best-matching prefix problem. Next, we show how to improve the performance of any best-matching prefix scheme using an initial array indexed by the first $X$ bits of the address. We then describe how to take advantage of cache line size to do a multiway search with six-way branching. Finally, we show how to extend the binary search solution and the multiway search solution for IPv6. For a database of $N$ prefixes with address length $W$, naive binary search would take $O(W * \log N)$; we show how to reduce this to $O(W+\log N)$ using multiple-column binary search. Measurements using a practical (Mae-East) database of 38000 entries yield a worst-case lookup time of $490 \mathrm{~ns}$, five times faster than the Patricia trie scheme used in BSD UNIX. Our scheme is attractive for IPv6 because of its small storage requirement ( $2 N$ nodes) and speed (estimated worst case of 7 cache line reads per lookup).
\end{abstract}

\section{INTRODUCTION}

$\mathbf{S}$ TATISTICS show that the number of hosts on the Internet is tripling approximately every two years [1]. Traffic on the Internet is also increasing exponentially. Traffic increase can be traced not only to an increasing number of hosts, but also to new applications (e.g., the Web, video conferencing, and remote imaging), which have higher bandwidth needs than traditional applications. One can only expect further increases in users, hosts, domains, and traffic. The possibility of a global Internet with multiple addresses per user (e.g., for appliances) has necessitated a transition from the older Internet routing protocol (IPv4 with 32-bit addresses) to the proposed nextgeneration protocol (IPv6 with 128-bit addresses).

Success opens up new sets of problems. As ordinary users begin to depend on the Internet, it is increasingly important that the Internet provide adequate performance. As a first step, communication links in the Internet backbone are being upgraded with high-speed fiber-optic links. However, even gigabit links are insufficient unless Internet routers are able to forward packets at gigabit rates. High-speed packet forwarding is compounded by increasing routing database sizes (due to increased number of hosts) and longer addresses (due to the transition to IPv6 [2], [3]). This paper deals with the problem of increasing IP packet-forwarding rates in routers. In par-

Manuscript received May 27, 1998; revised March 15, 1999; approved by IEEE/ACM TRANSACTIONS ON NETWORKING Editor C. Partridge

B. Lampson is with Microsoft Research, Seattle, WA 98122 USA (e-mail: blampson@microsoft.com).

V. Srinivasan and G. Varghese are with the Department of Computer Science, Washington University, St. Louis, MO 63130-4899 USA (e-mail: cheenu@ccrc.wustl.edu).

Publisher Item Identifier S 1063-6692(99)05707-6.
TABLE I

A Sample Routing Table

\begin{tabular}{l|c}
\hline Prefix & Next hop \\
\hline$*$ & L9 \\
$001^{*}$ & L1 \\
$0001^{*}$ & L2 \\
$011111^{*}$ & L3 \\
$100^{*}$ & L4 \\
$1000^{*}$ & L5 \\
$10001^{*}$ & L6 \\
\hline
\end{tabular}

ticular, we deal with a component of high-speed forwarding, address lookup, that is a major bottleneck.

When an Internet router gets a packet $P$ from an input link interface, it uses the destination address in packet $P$ to look up a routing database. The result of the lookup provides an output link interface, to which packet $P$ is forwarded. There is some additional bookkeeping, such as updating packet headers, but the major tasks in packet forwarding are address lookup and switching packets between link interfaces.

For gigabit routing, many solutions exist which do fast switching within the router box [4], [5]. Despite this, the problem of doing lookups at gigabit speeds remains. For example, Ascend's product [6] has hardware assistance for lookups and can take up to $3 \mathrm{~ms}$ for a single lookup in the worst case, and $1 \mathrm{~ms}$ on average. However, to support say $1 \mathrm{~Gb} / \mathrm{s}$ with an average packet size of 512 bits, lookups need to be performed in $500 \mathrm{~ns} /$ packet. Our scheme can be implemented in software on an ordinary PC in a worst-case time of $490 \mathrm{~ns}$, and so should be able to sustain a gigabit link even in the worst case.

The Best-Matching Prefix Problem: Address lookup can be done at high speeds if we are looking for an exact match of the packet destination address to a corresponding address in the routing database. Exact matching can be done using standard techniques such as hashing or binary search. Unfortunately, most routing protocols (including OSI and IP) use hierarchical addressing to avoid scaling problems. Rather than have each router store a database entry for all possible destination IP addresses, the router stores address prefixes that represent a group of addresses reachable through the same interface. The use of prefixes allows scaling to worldwide networks.

The use of prefixes introduces a new dimension to the lookup problem: multiple prefixes may match a given address. If a packet matches multiple prefixes, it is intuitive that the packet should be forwarded corresponding to the most specific prefix or longest matching prefix. IPv4 prefixes are arbitrary bit strings up to 32 bits in length, as shown in Table 1 . To see the difference between the exact matching and best-matching prefix, consider a 32-bit address $A$, whose first 8 bits are 
10000111. If we searched for $A$ in the above table, exact match would not give us a match. However, prefix matches are $100^{*}$ and $1000^{*}$, of which the longest matching prefix is $1000^{*}$, whose next hop is $L 5$.

The rest of this paper is organized as follows. Section II describes a model and the parameters used to evaluate schemes. Section III describes related work and briefly describes our contribution. Section IV contains our basic binary search scheme. Section $\mathrm{V}$ describes a basic idea of using an array as a front end to reduce the number of keys required for binary search. Section VI describes how we exploit the locality inherent in cache lines to do multiway search; we also describe measurements done using four publically available IPv4 databases. Section VIII describes how to do multicolumn and multiway search for IPv6. We also describe some measurements and projected performance estimates. Section IX states our conclusions.

\section{PERFormanCE MODEL}

The choice of a lookup algorithm depends crucially on assumptions about the routing environment and the implementation environment. We also need a performance model with precise metrics to compare algorithms.

\section{A. Routing Environment}

The Internet consists of local domains which are interconnected by a backbone consisting of multiple Internet service providers (ISP's). Accordingly, there are two interesting kinds of routers [7]: enterprise routers (used in a campus or organization) and backbone routers (used by ISP's). The performance needs of these two routers are different.

Backbone routers today [7] can have databases of up to 45000 prefixes (growing every day, several of them with multiple paths). The prefixes contain almost all lengths from 8 to 32; however, because of the evolution from Class B and Class $\mathrm{C}$ addresses, there is considerable concentration at 24- and 16-bit prefix lengths. Backbone routers typically run the border gateway protocol (BGP). Because some BGP implementations exhibit considerable instability, route changes can occur up to 100 times/s [7], [8]. This requires algorithms for handling route updates that take $10 \mathrm{~ms}$ or less. Notice here that we are assuming that two copies of the forwarding table are maintained, so that one copy can be used for forwarding while the other is being updated. Backbone routers may require frequent reprogramming, as ISP's attempt to deal with customer requirements such as virus attacks. The packet sizes seen are bimodal, and are typically either 45-byte control packets or 576-byte data packets.

Enterprise routers have smaller databases (up to 1000 prefixes) because of the heavy use of default routes for outside destinations. Routes are also typically much more stable, requiring route updates at most once every few seconds. The packet sizes are bimodal and are either 64 bytes or 1519 bytes. ${ }^{1}$

\footnotetext{
1 576-byte data packets arise in ISP's because of the use of a default size of 576 bytes for wide area traffic; 1519 hyte-size packets in the enterprise network probably arise from Ethernet maximum-size packets.
}

However, large multicampus enterprise routers may look more like backbone routers.

Address space depletion has lead to the proposal for the next generation of IP (IPv6) with 128-bit addresses. While there are plans for aggressive aggregation to reduce table entries, the requirement for both provider-based and geographical addresses, the need for connections to multiple ISP's, plans to connect control devices on the Internet, and the use of features like Anycast [2] all make it unlikely that backbone prefix tables will be smaller than in IPv4.

We use four publically available prefix databases for our comparisons. These are made available by the IPMA project [9] and are daily snapshots of the routing tables used at some major network access points (NAP's). The largest of these, Mae-East (about 38000 prefixes), is a reasonable model for a large backbone router; the smallest database, PAIX (around 713 prefixes), can be considered a model for an enterprise router. We will compare lookup schemes using these four databases with respect to three metrics: search time (most crucial), storage, and insert/delete times.

\section{B. Implementation Model}

We will consider both hardware and software platforms for implementing lookups. Software platforms are more flexible and have smaller initial design costs; hardware platforms have higher performance and are cheaper after volume manufacturing. For example, BBN [10] uses DEC Alpha CPU's in each line card, while Torrent and Rapid City [10] use hardwareforwarding engines.

Software: We will consider software platforms using modern processors such as the Pentium [11] and the Alpha [12]. These CPU's execute simple instructions very fast (few clock cycles) but take much longer to make a random access to main memory. However, if the data is in primary (L1) or secondary cache (L2), access times are only a few clock cycles. For example, when using an Alpha [12] processor, access to the on-chip L1 cache is 2 clock ticks, to on-chip L2 is 8 clock ticks, to off-chip L3 is around 16 ticks, and main memory is around 60 ticks.

The distinction arises because main memory uses slow cheap dynamic memory (DRAM, 60-100-ns access time), while cache memory is expensive but has fast static memory (SRAM, 10-20-ns latency). When a READ of a single word is made to memory, an entire cache line is fetched into the cache. This is important because the remaining words in the cache line can be accessed cheaply for the price of a single-memory READ.

Thus, a first-cut measure of the speed of any lookup algorithm, in either software or hardware, is the number of main memory (DRAM) accesses required, because these accesses often dominate search times. In some cases, SRAM may be used for off-chip memory. In both these cases, the number of off-chip memory accesses would dominate the search time. To count memory accesses, we must have an estimate of the total storage required by the algorithm to understand how much of the data structures can be placed in each of the memories. 
We chose a 300-MHz Pentium II (cost under \$2000) running Windows NT that has a 512-kbytes L2 cache and a cache line size of 32 bytes. We chose this platform because of the popularity of Wintel platforms, and the availability of useful tools. We believe the results would be similar if run on other comparable platforms such as the Alpha.

Hardware: To compare IP lookup schemes in hardware, we assume a cheap forwarding engine (say $\$ 20$, assuming high volumes) operating at a clock rate of $10 \mathrm{~ns}$. We assume the chip can place its data structure in SRAM (with $10 \mathrm{~ns}$ access times) and/or DRAM (60-100 ns access times). We assume, following current prices, that SRAM costs six times as much as DRAM per byte. While it possible to buy SRAM in small granularities, DRAM comes in large granularities (e.g., 2 Mbits). As in the software case, if we make a single READ to memory followed by subsequent READ's to adjacent locations, we can use page-mode DRAM to speed up the access to these adjacent locations. Thus, as in the software case, it pays to align data structures, so that memory references are made to adjacent locations.

Please note that in this paper, by memory reference we mean accesses to main memory. Cache hits are not counted as memory references. If a cache line of 32 bytes is read, then accessing two different bytes in the 32-byte line is counted as part of the same memory reference.

\section{PREvious WORK AND OUR CONTRIBUTIONS}

In this section, we review previous work on the longest matching prefix problem.

The current NetBSD implementation of IP lookup [13], [14] uses a Patricia trie, which processes an address one bit at a time. On a $200-\mathrm{MHz}$ Pentium with about 38000 entries in the routing table, this takes $1.5-2.5 \mu \mathrm{s}$ on average.

Many authors have proposed tries of high radix [15] but only for exact matching of addresses. OSI address lookups are done naturally using trie search four bits at a time [16]. This works because OSI prefix lengths are always multiples of four.

Schemes exist that can [17] do lookups in 200 ns using SRAM's (with $10 \mathrm{~ns}$ cycle times) to store the entire routing database. We note that large SRAM's are extremely expensive and are typically limited to caches in ordinary processors. Hardware solutions [18] based on content-addressable memories (CAM's) can be used to implement best-matching prefix: however, they are very expensive when large databases have to be supported.

Caching is a standard solution for improving average performance. However, experimental studies have shown poor cache hit ratios for backbone routers [17]. This is partly due to the fact that caches typically store whole addresses. Finally, schemes like tag switching [20], [21] and flow switching [17], [19] suggest protocol changes to avoid the lookup problem alltogether. These proposals depend on widespread acceptance, and do not completely eliminate the need for lookups at network boundaries.

In the last year, two new techniques [22], [23] for doing best-matching prefix have been announced. The approach in [22] is based on compressing trie nodes so that they will fit into the cache. The approach in [23] is based on doing binary search on the possible prefix lengths. Another form of compressed tries called LC tries are presented in [24].

Another approach based on prefix expansion [25] seems to be simple and fast. However, the technique that we present in this paper is attractive for IPv6 because of its bounded worstcase memory requirement. For IPv4, the binary search scheme presented in this paper would be more appropriate than other schemes for small enterprise routers that have less than 1000 prefixes. A comparison of these schemes can be found in [25].

Our Contributions: In this paper, we start by showing how to modify binary search to do best-matching prefix. ${ }^{2}$ Then we present multiway search, which improves the search speed, and multicolumn search, which is attractive for IPv6. We present measurements on the performance of all these schemes, and the improvements obtained by using an initial array.

Modified binary search requires two ideas: first, we treat each prefix as a range and encode it using the start and end of range; second, we arrange range entries in a binary search table and precompute a mapping between consecutive regions in the binary search table and the corresponding prefix.

Our approach is completely different from either [22] or [23], as we do binary search on the number of possible prefixes as opposed to the number of possible prefix lengths. However, we show that we can exploit the locality inherent in processor caches and fast cache line reads using SDRAM or RDRAM to do multiway search in $\log _{k+1} N+1$ steps, where $k>1$. We have found good results using $k=5$.

We also describe a simple scheme of using an initial array as a front end to reduce the number of keys required to be searched in binary search. The idea of using an initial array has been used in many implementations (e.g., [26]).

Measurements using the (Mae-East) database of 38000 entries yield a worst-case lookup time of $490 \mathrm{~ns}$, five times faster than the performance of the Patricia trie scheme used in BSD UNIX on the same database. We estimate a worstcase insertion time of around $300 \mathrm{~ms}$, which seems adequate for routing updates in an enterprise routing environment. We also estimate the performance of our scheme for IPv6 using a special SDRAM or RDRAM memory (which is now commercially available, though we could not obtain one to do actual experiments). This memory allows fast access to data within a page of memory, which enables us to speed up multiway search. Thus, in the worst case, we estimate seven cache line reads to search a large database of IPv6 entries.

\section{ADAPTING BINARY SEARCH FOR LONGEST MatChing PREFIX SEARCH}

Having described the model and performance measures, we now proceed to describe the main ideas behind our IP lookup scheme. The root idea behind our scheme is binary search. Recall, however, that binary search is typically used for exact match among fixed-length keys, while we need to find a longest match among variable-length prefixes. Thus,

\footnotetext{
${ }^{2}$ Our basic binary search technique has been described briefly in Perlman's book [16] but is due to the first author of this paper and has never been published before.
} 


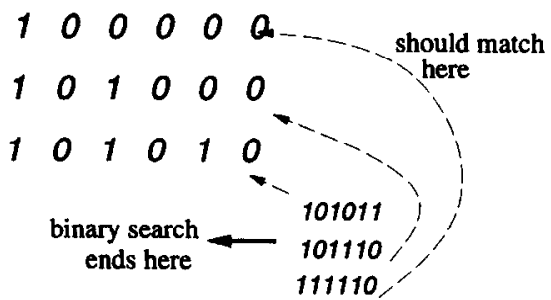

Fig. 1. Placing the three prefixes $1^{*}, 101^{*}$, and $10101^{*}$ in a binary search table by padding each prefix with zero's to make 6-bit strings and sorting the resulting strings. Note that the addresses 101011, 101110, and 111110 all end up in the same region in the binary search table (after the last entry), despite the fact that they have different longest matching prefixes.

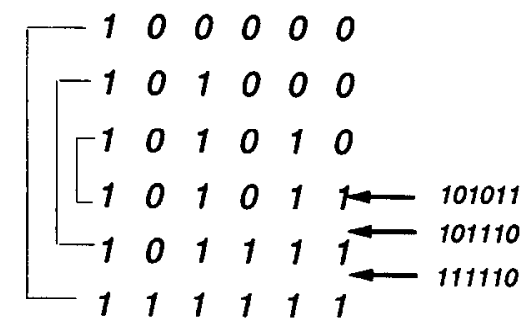

Fig. 2. We now encode each prefix in the table as a range using two values: the start and end of range. This time, the addresses that match different prefixes end up in different ranges.

adapting binary search to a longest matching prefix requires several subtle modifications.

To illustrate the progression of ideas, we use a simple contrived example, in which we have 6-bit addresses and just three prefixes: $1^{*}, 101^{*}$, and $10101^{*}$. Our first problem is that binary search does not work with variable-length strings. Thus, the simplest approach is to pad each prefix to be a 6-bit string by adding zeros (shown in Fig. 1).

Now consider a search for the three 6-bit addresses 101011, 101110 , and 111110. Since none of these addresses are in the table, binary search will fail. Unfortunately, on a failure, all three of these addresses will end up at the end of the table because all of them are greater than 101010, which is the last element in the binary search table. Notice, however, that each of these three addresses (see Fig. 1) has a different best-matching prefix.

Thus, we have two problems with naive binary search. First, when we search for an address, we end up far away from the matching prefix (potentially requiring a linear search). Second, multiple addresses that match to different prefixes end up in the same region in the binary table (Fig. 1).

\section{A. Encoding Prefixes as Ranges}

To solve the second problem, we recognize that a prefix like $1 *$ is really a range of addresses from 100000 to 111111 . Thus, instead of encoding $1^{*}$ by just 100000 (the start of the range), we encode it using both the start and end of range (100000 and 111111). Thus, each prefix is encoded by two full-length bit strings. These bit strings are then sorted. The result for the same three prefixes is shown in Fig. 2.

We connect the start and end of a range (corresponding to a prefix) by a line in Fig. 2. Notice how the ranges are nested. If we now try to search for the same set of addresses, they each

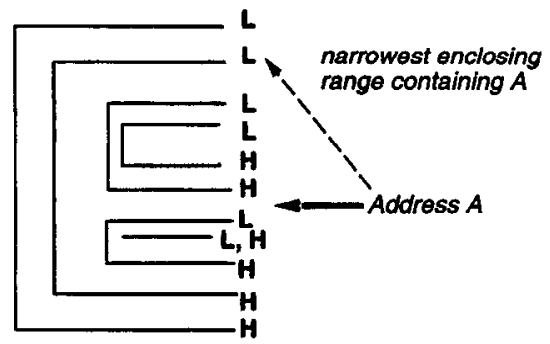

Fig. 3. Why longest matching prefix corresponds to narrowest enclosing range, and why each range in the modified binary search table maps to a unique prefix.

end in a different region in the table. To be more precise, the search for address 101011 ends in an exact match. The search for address 101110 ends in a failure in the region between 101011 and 101111 (Fig. 2), and the search for address 111110 ends in a failure in the region between 101111 and 111111. Thus, it appears that the second problem (multiple addresses that match different prefixes ending in the same region of the table) has disappeared. Compare Figs. 1 and 2.

To see that this is a general phenomenon, consider Fig. 3, which shows an arbitrary binary search table after every prefix has been encoded by the low (marked $\mathbf{L}$ in Fig. 3) and high points (marked $\mathbf{H}$ ) of the corresponding range. Consider an arbitrary position indicated by the solid arrow. If binary search for address $A$ ends up at this point, which prefix should we map $A$ to? It is easy to see the answer visually from Fig. 3 . If we start from the point shown by the solid arrow and go back up the table, the prefix corresponding to $A$ is the first $\mathbf{L}$ that is not followed by a corresponding $\mathbf{H}$ (see dotted arrow in Fig. 3.)

Why does this work? Since we did not encounter an $\mathbf{H}$ corresponding to this $\mathbf{L}$, it clearly means that $A$ is contained in the range corresponding to this prefix. Since this is the first such $\mathbf{L}$, this is the smallest such range. Essentially, this works because the best matching prefix has been translated to the problem of finding the narrowest enclosing range.

\section{B. Using Precomputation to Avoid Search}

Unfortunately, the solution depicted in Figs. 2 and 3 does not solve the first problem: notice that binary search ends in a position that is far away (potentially) from the actual prefix. If we were to search for the prefix (as described earlier), we could have a linear time search.

However, the modified binary search table shown in Fig. 3 has a nice property we can exploit. Any region in the binary search between two consecutive numbers corresponds to a unique prefix. As described earlier, the prefix corresponds to the first $\mathrm{L}$ before this region that is not matched by a corresponding $\mathbf{H}$ that also occurs before this region. Similarly, every exact match corresponds to a unique prefix.

Since this is the case, we can precompute the prefix corresponding to each region and the prefix corresponding to each exact match. This can potentially slow down insertion. However, the insertion or deletion of a new prefix should occur at a rate that is at least two orders of magnitude lower than packet-forwarding rates. Thus, slowing down insertion costs 


$\begin{array}{lllllllll}P 1) & 1 & 0 & 0 & 0 & 0 & 0 & P 1 & P 1 \\ P 2) & 1 & 0 & 1 & 0 & 0 & 0 & P 2 & P 2 \\ P 3) & 1 & 0 & 1 & 0 & 1 & 0 & P 3 & P 3 \\ 1 & 0 & 1 & 0 & 1 & 1 & P 2 & P 3 \\ 1 & 0 & 1 & 1 & 1 & 1 & P 1 & P 2 \\ 1 & 1 & 1 & 1 & 1 & 1 & - & P 1\end{array}$

Fig. 4. The final modified binary search table with precomputed prefixes for every region of the binary table. We need to distinguish between a search that ends in a success at a given point (= pointer) and search that ends in a failure at a given point ( $>$ pointer).

for the sake of faster forwarding is a reasonable tradeoff. (In Section VII-D, we show that insertion into even large databases can be done in several hundred milliseconds). Essentially, the idea is to add the dotted-line pointer shown in Fig. 3 to every region.

The final table corresponding to Fig. 3 is shown in Fig. 4. Notice that with each table entry $E$, there are two precomputed prefix values. If binary search for address $A$ ends in a failure at $E$, it is because $A>E$. In that case, we use the $>$ pointer corresponding to $E$. On the other hand, if binary search for address $A$ ends in a match at $E$, we use the $=$ pointer.

Notice that for an entry like 101011, the two entries are different. If address $A$ ends up at this point and is greater than 101011, clearly the right prefix is $P 2=101^{*}$. On the other hand, if address $A$ ends up at this point with equality, the correct prefix is $P 3=10101^{*}$. Intuitively, if an address $A$ ends up equal to the high point of a range $R$, then $A$ falls within the range $R$; if $A$ ends up greater than the high point of range $R$, then $A$ falls within the smallest range that encloses range $R$.

\section{Insertion into a Modified Binary Search Table}

The simplest way to build a modified binary search table from scratch is to first sort all the entries, after marking each entry as a high or low point of a range. Next, we process the entries using a stack, from the lowest to the highest to precompute the corresponding best-matching prefixes. Whenever we encounter a low point ( $L$ in the figures), we stack the corresponding prefix; whenever we see the corresponding high point, we unstack the prefix. Intuitively, as we move down the table, we are keeping track of the currently active ranges; the top of the stack keeps track of the innermost active range. The prefix on top of the stack can be used to set the $>$ pointers for each entry, and the = pointers can be computed trivially. This is an $O(N)$ algorithm if there are $N$ prefixes in the table.

One might hope for a faster insertion algorithm if we had to only add (or delete) a prefix. First, we could represent the binary search table as a binary tree in the usual way. This avoids the need to shift entries to make room for a new entry. Unfortunately, the addition of a new prefix can affect the precomputed information in $O(N)$ prefixes. This is illustrated in Fig. 5. The figure shows an outermost range corresponding to prefix $P$; inside this range are $N-1$ smaller ranges (prefixes) that do not intersect. In the regions not

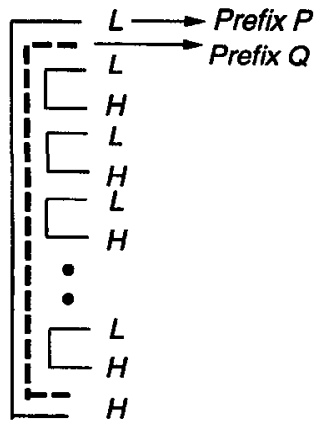

Fig. 5. Adding a new prefix $Q$ (dotted line) can cause all regions between an $\mathbf{H}$ and an $\mathbf{L}$ to move from Prefix $P$ to Prefix $Q$.

covered by these smaller prefixes, we map to $P$. Unfortunately, if we now add $Q$ (Fig. 5), we cause all these regions to map to $Q$, and $O(N)$ update process.

Thus, there does not appear to be any update technique that is faster than just building a table from scratch. Of course, many insertions can be batched; if the update process falls behind, the batching will lead to more efficient updates. We will see shortly that using an initial 16-bit table lookup can decompose the single binary search table into multiple (but smaller) binary search tables. Since the decomposed binary search tables are smaller, this decomposition not only speeds up search time but also speeds up insertion. We defer estimates of insertion times to Section VII-D. First, we describe two crucial optimizations that considerably speed up the basic binary search scheme.

\section{Using A Precomputed 16-Bit Prefix Table}

We can improve the worst-case number of memory accesses of the basic binary search scheme with a precomputed table of best-matching prefixes for the first $X$ bits. This idea has been used in many routers. The best-matching prefixes of the elements of the array can be precomputed using a form of prefix expansion [25].

The main idea is to effectively partition the single binary search table into multiple binary search tables for each value of the first $X$ bits. We choose $X=16$ for what follows, as the table size is about as large as we can afford, while providing maximum partitioning.

The best-matching prefixes for the first 16-bit prefixes can be precomputed and stored in a table. This table would then have $M a x=65536$ elements. For each index $X$ of the array, the corresponding array element stores best-matching prefix of $X$. Additionally, if there are prefixes of longer length with that prefix $X$, the array element stores a pointer to a binary search table/tree that contains all such prefixes. Insertion, deletion, and search in the individual binary search tables are identical to the techniques described earlier in Section IV.

Without the initial table, the worst-case possible number of memory accesses is $\log _{2} N+1$, which for large databases could be 16 or more memory accesses. For a sample database, this simple trick of using an array as a front end reduces the maximum number of prefixes in each partitioned table to 336 from the maximum value of over 38000 , which leads to a worst case of 10 memory accesses. 


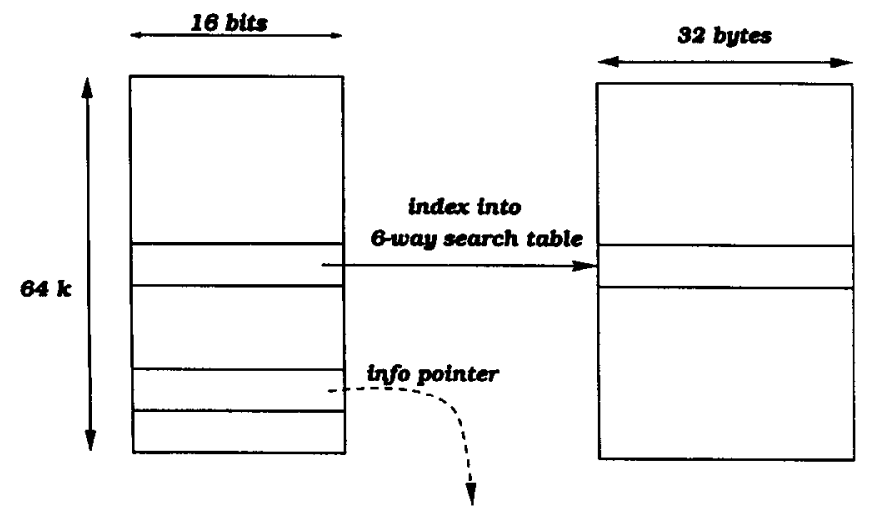

Fig. 6. The initial 16-bit array, with pointers to the corresponding six-way search nodes.

\section{Multiway Search: Exploiting the Cache Line}

Modern processors have wide cache lines. The Intel Pentium Pro has a 64-bit data bus and a cache line size of 32 bytes. Main memory is usually arranged in a matrix form, with rows and columns. Accessing data given a random row address and column address has an access time of 50-60 ns. However, using SDRAM or RDRAM, filling a cache line of 32 bytes (which is a burst access to four contiguous 64-bit DRAM locations) is much faster than accessing four random DRAM locations. When accessing a burst of contiguous columns in the same row, while the first piece of data would be available only after $60 \mathrm{~ns}$, further columns would be available much faster. SDRAM's (synchronous DRAM's) are available (at less than $\$ 100$ for 8 Mbits) that have a column access time of $10 \mathrm{~ns}$. Timing diagrams of micron SDRAM's are available through [27]. RDRAM's [28] are available that can fill a cache line in $101 \mathrm{~ns}$. Detailed descriptions of main memory organization can be found in [29].

The significance of this observation is that it pays to restructure data structures to improve locality of access. To make use of the cache line fill and the burst mode, keys and pointers in search tables can be laid out to allow multiway search instead of binary search. This effectively allows us to reduce the search time of binary search from $\log _{2} N$ to $\log _{k+1} N$, where $k$ is the number of kcys in a scarch nodc. The main idea is to make $k$ as large as possible so that a single search node (containing $k$ keys and $2 k+1$ pointers) fits into a single cache line. If this can be arranged, an access to the first word in the search node will result in the entire node being prefetched into cache. Thus, the accesses to the remaining keys in the search node are much cheaper than a memory access.

We did our experiments using a Pentium Pro; the parameters of the Pentium Pro resulted in us choosing $k=5$ (i.e., doing a six-way search). For our case, if we use $k$ keys per node, then we need $2 k+1$ pointers, each of which is a 16-bit quantity. (This is because we need a pointer for the ranges between keys, as well as a pointer for exact matches with each key.) Thus, in 32 bytes, we can place five keys and hence can do a six-way search. The initial full array of 16 bits followed by the six-way search is depicted in Fig. 6.

Since the worst case (for the Mae-East database after using a 16 bit initial array) has 336 entries in a table, this leads to a worst case of four memory accesses (since $6^{4}=1296$ takes only four memory accesses when doing a six-way search), in addition to the initial table lookup.

\section{A. Search}

The following search procedure can be used for both $1 \mathrm{Pv} 4$ and IPv6 (see next section for details on the IPv6 search procedure).

1) Index into the first 16-bit array using the first 16 bits of the address.

2) If the pointer at the location is an information pointer, return it. Otherwise enter the six-way search with the initial node given by the pointer, and the key being the next 16 bits of the address.

3) In the current six-way node locate the position of the key among the keys in the six-way node. We use binary search among the keys within a node. If the key equals any of the keys keyi in the node, use the corresponding pointer $p t r_{i}$. If the key falls in a range formed by the keys, use the pointer $p t r_{i, i+1}$. If this pointer is an information pointer, return it; otherwise repeat this step with the new six-way node given by the pointer.

In addition, we allow multicolumn search for IPv6 (see Section VIII) as follows. If we encounter an equal to pointer, the search shifts to the next 16 bits of the input address. This feature can be ignored for now, and will be understood after reading Section VIII.

As the data structure itself is designed with a node size equal to a cache line size, good cacheing behavior is a consequence. All the frequently accessed nodes will stay in the cache. To reduce the worst-case access time, the first few levels in a worst-case depth tree can be cached.

\section{MEasurement AND COMPARISONS FOR IPv4}

We used a 200-MHz Pentium Pro-based [30] machine (cost under $\$ 2000$ ) with a 8 -kbytes four-way set-associative primary instruction cache and an 8-kbytes dual-ported two-way set associative primary data cache. The L2 cache is 256 kbytes of SRAM that is coupled to the core processor through a full clock-speed 64-bit cache bus.

We used practical routing tables (primarily Mae-East with over 38000 entries, all of which were obtained from [9]) for our experiments. Our tables list results for the BSD Patricia trie implementation (extracted from the BSD kernel into user space), basic binary search, binary search with a 16-bit initial array, and six-way search.

\section{A. Worst-Case Lookup Times}

After adding the routes in the route database [9], several IP addresses were generated and a lookup performed 10 million times for each such address. The lookup time for an address was then calculated by taking the total elapsed time and (measured by reading the clock before and after the iterations) dividing it by the number of iterations ( 10 million). As one would expect, the numbers vary depending on the number of levels of the binary tree that must be traversed before the prefix of the address is resolved. 
TABLE II

Time Taken for Single Address Lookup on a Pentium Pro

\begin{tabular}{r|r|c|r}
\hline Patricia & $\begin{array}{r}\text { Basic } \\
\text { binary } \\
\text { search }\end{array}$ & $\begin{array}{r}16 \text { bit } \\
\text { +binary } \\
\text { search }\end{array}$ & $\begin{array}{r}16 \text { bit } \\
+6 \text { way } \\
\text { search }\end{array}$ \\
\hline Time & Time & Time & Time \\
(nsec) & $($ nsec) & (nsec) & (nsec) \\
\hline 1530 & 1175 & 730 & 490 \\
1525 & 990 & 620 & 490 \\
1450 & 1140 & 470 & 390 \\
2585 & 1210 & 400 & 300 \\
1980 & 1440 & 330 & 210 \\
810 & 1220 & 90 & 95 \\
1170 & 1310 & 90 & 90 \\
\hline
\end{tabular}

Several addresses were searched and the search times noted. Shown in the table are addresses picked to illustrate the variation in time of the 16-bit initial table+six-way search method. Thus, the first two rows correspond to the maximum depth of the search tree, while the last two rows correspond to the minimum depth (i.e., no prefixes in search table).

For example, in the six-way search scheme, any address whose prefix is less than 16 bits will be resolved in one array lookup (depth 0 ); an address will take one more access to a sixway node to resolve (depth 1) if the number of keys starting with the 16-bit prefix of the address is less than 5; others will take accesses to a second six-way node if the number of keys starting with the 16-bit prefix of the address is less than 35 (depth 2), and so on. Recall that as we are doing six-way search, the depth of an address $A$ is equal to $\left\lceil\log _{6} n(A)\right\rceil$, where $n(A)$ is the number of keys that start with the 16-bit prefix of address $A$. Since we have seen that $n(A) \leq 336$ for Mae-East, the depth of an address is no greater than 4 in our experiments.

Since the worst-case lookup times varied with depth, we picked seven address lookups that would end at different depths to display in Table II. For example, the first two rows corresponds to Depth 4 addresses, while the last two correspond to Depth 0 addresses. The third, fourth, and fifth rows correspond to Depth 1, Depth 2, and Depth 3 addresses, respectively. Table II compares the lookup times for these seven addresses for each of four schemes: Patricia (baseline), basic binary search, binary search with the initial array, and six-way search. The time for Patricia does vary a great deal (from around 1.5-2.5 ms). The search time for basic binary search varies only a little (from around $1-1.3 \mathrm{~ms}$ ). The time for binary search with the initial table clearly depends on the depth of the address. The lower rows have very small lookup times ( $90 \mathrm{~ns}$ ), but the upper rows have larger times (e.g., 730 ns) corresponding to a binary search of up to 336 keys.

The time taken for the six-way search varies in the same way. The lookup times for depth 0 nodes are nearly identical for six-way and binary search +16-bit table because both involve only a lookup of the initial array. However, the times at each depth greater than depth 0 are much lower than that of using binary search + the 16-bit table because of the benefits of using six-way versus two-way search. Recall that we have defined depth of an address with respect to the six-way scheme. An address at depth 4 in the six-way scheme will be at a greater depth in the other binary search schemes.

Notice also that in Table II that the lookup times for six-way search increase by around $100 \mathrm{~ns}$ for each memory access,
TABLE III

Memory REquirement and Worst-Case Time

\begin{tabular}{l|c|c|c|c}
\hline & $\begin{array}{c}\text { Patr } \\
\text { icia }\end{array}$ & $\begin{array}{c}\text { Basic } \\
\text { Binary } \\
\text { Search }\end{array}$ & $\begin{array}{c}16 \text { bit } \\
\text { table } \\
\text { tbinary }\end{array}$ & $\begin{array}{c}16 \text { bit } \\
\text { table } \\
\text { +6 way }\end{array}$ \\
\hline $\begin{array}{l}\text { Mem for } \\
\text { building(MB) }\end{array}$ & 3.2 & 3.6 & 1 & 1 \\
\hline $\begin{array}{l}\text { Mem for } \\
\text { searchable } \\
\text { structurc (MB) }\end{array}$ & 3.2 & 1 & 0.5 & 0.7 \\
\hline $\begin{array}{l}\text { Worst case } \\
\text { search(nsec) }\end{array}$ & 2585 & 1310 & 730 & 490 \\
\hline $\begin{array}{l}\text { Worst case } \\
\text { faster than } \\
\text { Patricia by }\end{array}$ & 1 & 2 & 3.5 & 5 \\
\hline
\end{tabular}

yielding a lookup time of around $100 *(D(A)+1)$ for an address $A$, where $D(A)$ is the depth of address $A$ in the six-way scheme. This fits in well with our intuition that the primary measure of lookup speed is the number of memory accesses, and that the speed of a random memory access (including memory costs, processing costs and costs of cache misses) in the Pentium is around $100 \mathrm{~ns}$.

Instruction count: The static instruction count using a full 16-bit initial table followed by a six-way search table is less than 100 instructions on the Pentium Pro.

\section{B. Memory Requirements and Worst-Case Times}

We have seen that six-way search improves the worst-case time to $490 \mathrm{~s}$. What is the storage penalty for this extra speed? To answer this question, we used the Mae-East database and compared the three schemes (basic binary search, binary search with 16-bit initial table, and binary search with 16-bit initial table plus six-way search) in Table III. We also used the Patricia trie code (that we extracted from the BSD code) as a baseline comparison.

Table III shows that six-way search takes a worst-case time of $490 \mathrm{~ns}$ (compared to 2585 ns for Patricia and 1310 ns for ordinary binary search) and, surprisingly, has a memory usage (0.95 Mbits for search structure) that is less than Patricia (3.2 Mbits) and even less than for basic binary search (1 Mbits). The fact that the memory for the binary search with an initial array is less than that for basic binary search appears counterintuitive. The explanation is that while the keys used in the regular binary search are 32 bits and the pointers involved are also 32 bits, in the 16-bit table followed by the six-way search case, both the keys and the pointers are 16 bits. This is possible because the initial 16-bit lookup allows us to work with 16-bit quantities after the lookup. The use of smaller sized quantities is only partially offset by the size of the added initial table.

From Table III, we can see that the initial array improves the performance of the binary search from a worst case of 1310-730 ns; multiway search further improves the search time to 490 ns. Thus, both optimizations (initial array, multiway search) appear to be worthwhile.

\section{Variation of Lookup Times and Memory with Database Size}

There are at least three other databases in [9] besides the large Mae-East database. The Mae-East database may be characteristic of a router used by a large service provider. 
TABLE IV

Memory Requirement, Worst-Case Time, and Build Times For Four Prefix Databases Using Six-Way SEARCh

\begin{tabular}{c|c|c|c|c}
\hline & MaeEast & MaeWest & Pac & Paix \\
\hline Number of Prefixes & 38816 & 14072 & 3812 & 713 \\
\hline Worst Case Search Time(nsec) & 490 & 490 & 390 & 300 \\
\hline Memory Required (kbytes) & 950 & 512 & 530 & 265 \\
\hline Build Time (seconds) & 5.8 & 2.16 & 0.78 & 0.35 \\
\hline
\end{tabular}

The other databases (Mae-West, Pac, and Paix) may reflect more common database sizes. In particular, the smallest Paix database may be an accurate model of a large enterprise router in a campus or a company.

Table IV shows the number of prefixes, the worst-case search times, and the memory requirements for the four databases using six-way search. Notice that the storage goes down from 950 kbytes to around 265 kbytes for the smallest database (small enough to fit into cache). Also notice that the worst-case lookup time goes down (though not dramatically) from 490 (Mae-East) to $300 \mathrm{~ns}$ (Paix). This is because the worst-case tree depth often decreases as we reduce the number of prefixes.

We note that for the Paix database, the storage is completely dominated by the initial 16-bit array ( 250 kbytes out of a total of 265 kbytes). This suggests that for such small databases, we should use a smaller initial array (e.g., 12 bits) which can reduce storage greatly at the potential cost of a small increase in worst-case tree depth. Picking the optimal (or near-optimal) initial array size for each database is an interesting possibility that we have not yet explored.

\section{Building and Incremental Insertion Times}

So far, we have ignored insertion and table-building times. The heavy use of precomputation in the basic binary search scheme suggests that insertion times can be quite slow. While many existing schemes suggest that lookup times are far more important than insertion times [22], [23], the existence of rapid BGP updates in the backbone makes rapid insertion (and deletion) an issue, at least for backbone routers (though not for enterprise routers).

Table IV also lists the time to build the complete search structure for six-way search for each of the four databases. Wc made no attempt to optimize the table-building process; it seems very likely that the numbers can be improved. From the table, we see that the cost of building a complete table can range from $5.8 \mathrm{~s}$ (for Mae-East) to $350 \mathrm{~ms}$ (for Paix). At first glance, this seems to preclude the use of binary search for large backbone routers because of the potential need for rapid insertion times (10-100 ms). However, incremental insertion times can be much smaller, even for large databases like Mae-East.

The worst-case insertion/deletion cost can be estimated as follows. For prefixes of size less than 16 bits, we may have to scan the entire initial table to update any table entries that may be affected by this prefix's insertion or deletion. This will potentially require scanning $2^{16}$ trie-node entries. On a Pentium with a Write Back cache, while the first word in a cache line would cost $100 \mathrm{~ns}$ for the read, the writes would be only to cache and the entire cache line will take at most $200 \mathrm{~ns}$ to update. A single cache line holds ten entries, so at 20 ns/entry for $2^{16}$ entries, we get $1.25 \mathrm{~ms}$.

For a prefix $P$ of greater than 16 bits, we simply have to insert/delete $P$ from the binary search tree corresponding to the 16-bit prefix of $P$. Our results indicate that even for Mae-East, the worst-case number of keys corresponding to a 16-bit initial prefix is at most 336 . Thus, we can estimate the insertion/deletion time (looking at the worst-case building time for the multiway search structure for the Paix database) to be no more than $352 \mathrm{~ms}$. In practice, the average insertion time should be much faster because: 1) the individual search trees are typically much smaller than the worst case of $336 ; 2$ ) our estimates are very conservative; and 3) we have made no effort to optimize insertion costs. An average insertion time of around $20 \mathrm{~ms}$ seems easily achievable even for very large databases like Mae-West. Note that the technique of using an initial array actually speeds up incremental insertion because it replaces a single large binary tree (into which insertion/deletion can be expensive) by several smaller binary trees (into which insertion/deletion is cheap).

\section{E. Comparing Six-Way Search to Other Algorithms}

So far, we have been using the Patricia trie code extracted from NetBSD as a baseline comparison. However, this is not really a fair comparison for the following reasons. First, the NetBSD code [14] uses backtracking, which slows down the code, and can be removed by simple optimizations. Second, the NetBSD code performs other functions besides basic IP lookup, which may increase lookup times and increase memory usage. However, in the last two years, there have been a number of other algorithms that have attracted considerable attention. These include binary search on hash tables [23], the Lulea scheme for compressed multibit tries [22], the levelcompressed trie scheme (LC tries) [24], and multibit tries using prefix expansion [25].

While we have not implemented all these schemes, we were fortunate that the two schemes we have not implemented (LC tries and Lulea scheme) have been implemented by their inventors on a comparable Pentium platform using the same Mae-East database. This allows us to do a reasonable comparison of all five schemes. We borrow the numbers for binary search on hash tables and multibit tries from our earlier paper [25]. The numbers for LC tries are taken from [24], and the numbers for the Lulea scheme are taken from [22].

The comparison is not without flaws; since the Mae-East database is constantly changing, and the reported measurements were done based on different snapshots, the number of prefixes is not exactly the same (but is roughly 40000 prefixes) in all measurements. The Lulea scheme was implemented and measured on a $200-\mathrm{MHz}$ Pentium, the LC trie on a $133-\mathrm{MHz}$ Pentium, and the multibit trie scheme using a 300$\mathrm{MHz}$ Pentium. In those cases, we have (somewhat generously) scaled the schemes that proportionately to a common 300$\mathrm{MHz}$ clock speed used in [25]. This is rather generous, because scaling up the clock speed does not necessarily increase lookup times by the same factor, because memory access times do 
TABLE V

Comparing the Worst-Case Lookur Times for AdDress Matcilis

\begin{tabular}{l|r|r|r}
\hline & $\begin{array}{r}\text { Worst case for } \\
24 \text { bit prefix } \\
\text { (nsec) }\end{array}$ & $\begin{array}{r}\text { Worst } \\
\text { case } \\
\text { (nsec) }\end{array}$ & $\begin{array}{r}\text { Memory required for } \\
\text { Mae East database } \\
\text { (KBytes) }\end{array}$ \\
\hline \hline Patricia trie & 1000 & 1650 & 3262 \\
\hline $\begin{array}{l}\text { 6-way search } \\
\text { on prefixes }\end{array}$ & 330 & 330 & 950 \\
\hline $\begin{array}{l}\text { Binary search } \\
\text { on hash tables }\end{array}$ & 250 & 650 & 1600 \\
\hline Lulea scheme & 349 & 409 & 160 \\
\hline Multibit trie & 180 & 236 & 640 \\
\hline LC tries & 1000 & - & 700 \\
\hline
\end{tabular}

Note that numbers measured on $200-$ and $133-\mathrm{MHz}$ pentiums have been projected to $300 \mathrm{MHz}$.

not speed up with the faster clock. Despite these limitations, we believe such a comparison is still useful as an initial study.

Table $\mathrm{V}$ describes a comparison of the various schemes in terms of lookup times and memory usage. Many implementors believe that while the absolute worst-case times are interesting, since almost all prefixes are 24 bits or less in length, the worst-case time to resolve 24-bit prefixes is an important metric. This figure is especially favorable to trie schemes that can terminate faster (i.e., at a smaller trie depth) when the best-matching prefix is 24 bits or less. Thus, we also have a column corresponding to the worst case lookup times for 24bit prefixes. The numbers for Patricia trie and six-way search have been scaled to $300 \mathrm{MHz}$, which is why they differ from the numbers reported earlier. The numbers for Lulea and LC tries have also been scaled similarly from 133 and $200 \mathrm{MHz}$, respectively.

We note that the LC and Patricia trie implementations are fairly slow, but the memory needed for LC tries is much smaller than Patricia tries. (The worst-case times for LC tries are not reported in [24].) The memory for the Lulea scheme is easily the smallest (160 kbytes) and multibit tries have the fastest lookup times (180 ns). Six-way search does reasonably well, with 950 kbytes of memory and a lookup time (projected to $300 \mathrm{MHz}$ ) of $330 \mathrm{~ns}$.

An important factor not shown in the table is the cost of insertion and deletion of prefixes. This is not described in the papers on LC tries, binary search on hash tables, or Lulea compressed tries. Thus, a complete comparison is not possible. Since the Lulea, LC trie, and binary search on hash table schemes all potentially require changing the complete data structure on a prefix insertion/deletion, they are likely to have slow insertion/deletion times. The multibit trie scheme estimates insertion/deletion at $2.5 \mathrm{~ms}$. Recall that we estimated an insertion deletion time of around $350 \mathrm{~ms}$ for six-way search.

Based on the comparison, the Lulea scheme appears to be the candidate of choice if small memory (useful especially if SRAM is used for memory and cost is an issue) is the dominant factor. The multibit trie scheme seems to have the best lookup and insertion times, while allowing reasonably small memory. However, we note that the memory usage of the multibit trie schemes is only small when a complicated dynamic programming algorithm is used to lay out the variable stride trie [25]. This algorithm may need to be run only rarely, but adds complexity and can slow down insertion and deletion when it is run.

Recall from the introduction that there are two types of routcrs: backbone and enterprise. Enterprise routers have small databases and have less stringent requirements in terms of route changes. For such routers and small databases, six-way search is simple and fast (see earlier results for the Paix database) and has adequate insertion and deletion times. Thus, we believe that six-way search is a reasonable candidate for implementation in an Enterprise router.

\section{Multiway AND MUlticolumn SEARCH}

In this section, we describe the problems of searching for identifiers of large width (e.g., 128-bit IPv6 address or 20-byte OSI addresses). We first describe the basic ideas behind multicolumn search and then proceed to describe an implementation for IPv6 that uses both multicolumn and multiway search. We then describe sample measurements using randomly generated IPv6 addresses.

\section{A. Multicolumn Binary Search of Large Identifiers}

The scheme we have described in earlier sections can be implemented efficiently for searching 32-bit IPv4 addresses. Unfortunately, a naive implementation for IPv6 can lead to inefficiency. Assume that the word-size $M$ of the machine implementing this algorithm is 32 bits. Since IPv6 addresses are 128 bits (four machine words), a naive implementation would take $4 \cdot \log _{2}(2 N)$ memory accesses. For a reasonably sized table of around 32000 entries, this is around 60 memory accesses! Six-way search does not help this problem (entries after the initial 16-bit table need only be 112 bits long, but that still requires four machine words.)

In general, suppose each identifier in the table is $W / M$ words long (for IPv6 addresses on a 32-bit machine, $W / M=$ 4). Naive binary search will take $W / M \cdot \log N$ comparisons, which is expensive. Yet, this seems obviously wasteful. If all the identifiers have the same first $W / M-1$ words, then clearly $\log N$ comparisons are sufficient. We show how to modify binary search to take $\log N+W / M$ comparisons. It is important to note that this optimization we describe can be useful for any use of binary search on long identifiers, not just the best-matching prefix problem.

The strategy is to work in columns, starting with the most significant word and doing binary search in that column until we get equality in that column. At that point, we move to the next column to the right and continue the binary search where we left off. Unfortunately, this does not quite work.

In Fig. 7, which has $W / M=3$, suppose we are searching for the three-word identifier $B M W$ (pretend each character is a word). We start by comparing, in the left-most column, the middle element (shown by the arrow labeled 1). Since the $B$ in $B M W$ matches the $B$ at the arrow labeled 1 , we move to the right (not shown) and compare the $M$ in $B M W$ with the $N$ in the middle location of the second column. Since $N<M$, we do the second probe at the quarter position of the 


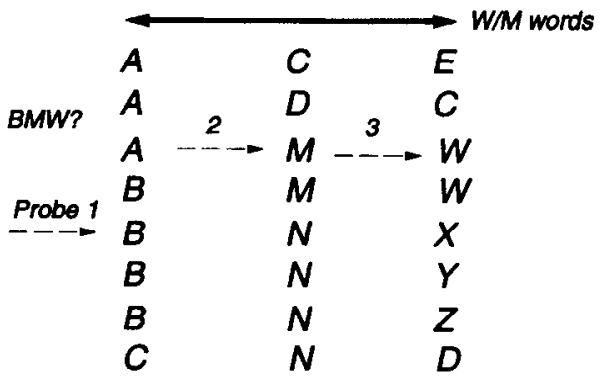

Fig. 7. Binary search by columns does not work when searching for $B M W$.

second column. This time, the two $M$ 's match and we move to the right and find $W$; but we have found $A M W$, not $B M W$, which we were looking for.

The problem is caused by the fact that when we moved to the quarter position in column 2 , we assumed that all elements in the second quarter begin with $B$. This assumption is false in general. The trick is to add state to each element in each column which can contain the binary search to stay within a guard range.

In the figure, for each word like $B$ in the left-most (most significant) column, we add a pointer to the range of all other words that also contain $B$ in this position. Thus, the first probe of the binary search for $B M W$ starts with the $B$ in $B N X$. On equality, we move to the second column as before. However, we also keep track of the guard range corresponding to the $B$ 's in the first column. The guard range (rows 4 through 6) is stored with the first $B$ we compared.

Thus, when we move to column 2 and we find that $M$ in BMW is less than the $N$ in $B N X$, we attempt to halve the range as before, and try a second probe at the third entry (the $M$ in $A M W$ ). However, the third entry is lower than the high point of the current guard range (4-6). So, without doing a comparison, we try to halve the binary search range again. This time we try entry 4 , which is in the guard range. We get equality and move to the right, and find $B M W$ as desired.

In general, every multiword entry $W_{1}, W_{2}, \cdots, W_{n}$ will store a guard range with every word. The range for $W_{i}$ points to the range of entries that have $W_{1}, W_{2}, \cdots, W_{i}$ in the first $i$ words. This ensures that when we get a match with $W_{i}$ in the $i$ th column, the binary search in column $i+1$ will only search in this guard range. For example, the $N$ entry in $B N Y$ (second column) has a guard range of 5-7, because these entries all have $B N$ in the first two words.

The resulting search strategy takes $\log _{2} N+W / M$ probes if there are $N$ identifiers. The cost is the addition of two 16-bit pointers to each word. Since most word sizes are at least 32 bits, this results in adding 32 bits of pointer space for each word, which can at most double the memory usage. Once again, the dominant idea is to use precomputation to trade a slower insertion time for a faster search.

We note that the whole scheme can be elegantly represented by a binary search tree with each node having the usual $>$ and $<$ pointers, but also an $=$ pointer, which corresponds to moving to the next column to the right, as shown above. The subtree corresponding to the $=$ pointer naturally represents the guard range.

\section{B. Using Multicolumn and Multiway Search for IPv6}

In this section, we explore several possible ways of using the k-way search scheme for IPv6. We saw that for IPv4, the maximum number in a single tree was 336 for a practical database with $N$ more than 38000 (i.e., the number of prefixes that have the same first 16 bits is 168 , leading to 336 keys). For IPv6, even if there is an increase of ten times in the number of prefixes that share the same first 16 bits, for 2048 prefixes in a tree, we get a worst case of nine cache line fills with a 32-byte cache line. For a 64-bytc cache line machine, we get a worst case of seven cache line fills. This would lead to worst-case lookup times of less than $800 \mathrm{~ns}$, which is competitive with the scheme presented in [23].

\section{Measurements}

We generated random IPv6 prefixes and inserted them into a k-way search with an initial 16-bit array. Clearly, the use of a randomly generated IPv6 address database is no substitute for measurements with actual IPv6 databases. (In particular, random generation will make the use of the 16-bit tablc appear beneficial. However, it is likely that IPv6 addresses will initially consist of IPv4 addresses padded with a constant prefix. Such an address assignment policy would cause the initial 16 bit table to do badly.) However, until realistic databases become available, randomly generated address databases are a reasonable method to evaluate algorithms.

From the Mae-East database, it was seen that with $N$ of about 38000 , the maximum number which shared the first 16 bits was about 300 , which is about $1 \%$ of the total number of prefixes. To capture this, when generating IPv6 prefixes, we generated the last 112 bits randomly and distributed them among the slots in the first 16-bit table, such that the maximum number that falls in any slot is around 1000 . This is necessary because if the whole IPv6 prefix is generated randomly, even with $N$ about 60000 , only one prefix will be expected to fall in any first 16-bit slot. For this distribution, on a Pentium Pro which has a cache line of 32 bytes, the worst-case search time was found to be $970 \mathrm{~ns}$.

\section{CONCLUSION}

We have described a basic binary search scheme for the longest matching prefix problem. Basic binary search requires two new ideas: encoding a prefix as the start and end of a range, and precomputing the best-matching prefix associated with a range. We have then presented three crucial enhancements: 1) use of an initial array as a front end; 2) multiway search; and 3) multicolumn search for long addresses.

We have shown how using an initial precomputed 16-bit array can reduce the number of required memory accesses from 16 to 9 in a typical database; we expect similar improvements in other databases. We then presented the multiway search technique, which exploits the fact that most processors prefetch an entire cache line when doing a memory access. A six-way branching search leads to a worst case of five cache line fills in a Pentium Pro which has a 32-byte cache line. We presented measurements for IPv4. Using a typical database of over 38000 prefixes, we obtain a worst-case time of $490 \mathrm{~ns}$, an 
average time of $100 \mathrm{ns,} \mathrm{storage} \mathrm{of} 0.95$ Mbits, and estimated insertion times of around $350 \mathrm{~ms}$. For smaller databases (less than 1000 prefixes), we obtain search times of around 300 ns and storage of 265 kbytes. The simplicity of the scheme makes it attractive for use in enterprise routers that have small routing tables.

For IPv6 and other long addresses, we introduced a multicolumn search that avoided the multiplicative factor of $W / M$ inherent in basic binary search by doing binary search in columns of $M$ bits, and moving between columns using precomputed information. We have estimated that this scheme potentially has a worst case of 7 cache line fills for a database with over 50000 IPv6 prefixes.

For future work, we are considering the problem of using different numbers of bits in each column of the multicolumn search. We are also considering the possibility of laying out the search structure to make use of the page-mode load to the L2 cache by prefetching. Another possible extension is to retrofit our Pentium Pro with an SDRAM or RDRAM to improve cache loading performance; this should allow us to obtain better measured performance.

\section{REFERENCES}

[1] M. Gray. (1996). Internet Growth Summary. [Online]. Available HTTP: http://www.mit.edu/people/mkgray/net/internet-growth-summary.html

[2] S. Deering and R. Hinden. (1995). Internet Protocol. Version 6 (IPv6), Specification RFC 1883, IETF. [Online]. Available HTTP: http://www.ietf.org/rfc/

[3] C. Huitema, IPv6: The New Internet Protocol. Englewood Cliffs, NJ: Prentice-Hall, 1996.

[4] N. McKeown, M. Izzard, A. Mekkittikul, B. Ellersick, and M. Horowitz, "The tiny tcra: A packet switch core," IEEE Micro, vol. 17, no. 1, Jan. 1997.

[5] J. Turner, "Design of a gigabit ATM switch," presented at the IEEE INFOCOM'97, Kobe, Japan.

[6] Ascend GRF IP switch, Ascend Corporation, Alameda, CA, 1996.

[7] S. Bradner, "Next generation routers overview," presented at the Networld Interop' 97, Atlanta, GA.

[8] C. Labovitz, G. Malan, and F. Jahanian, "Internet routing instability," presented at the SIGCOMM'97, Cannes, France.

[9] Merit. (1997). "Routing table snapshot on 14 Jan 1997 at the Mae-East NAP." [Online]. Available FTP: ftp://merit.edu/statistics/ipma

[10] C. Partridge et al., "A 50-Gb/s IP router," IEEE/ACM Trans. Networking, vol. 6, pp. 237-248, June 1998.

[11] Pentium Pro and Pentium II Processors and Related Products, Intel Corp., Santa Clara, CA, 1998.

[12] R. Sites and R. Witek, Alpha AXP Architecture Reference Guide. London, U.K.: Butterworth, 1995.

[13] R. Stevens and G. Wright, TCP/IP Illustrated-Vol. 2: The Implementation. Reading, MA: Addison-Wesley, 1995.

[14] K. Sklower, "A tree-based routing table for Berkeley unix," presented at the 1991 Winter Usenix Conf., Dallas, TX.

[15] T. Pei and C. Zukowski, "Putting routing tables in silicon," IEEE Network Mag., Jan. 1992.

[16] R. Perlman, Interconnections, Bridges und Routers. Reading, MA: Addison-Wesley, 1992.

[17] P. Newman, G. Minshall, and L. Huston, "IP switching and gigabit routers," IEEE Commun. Mag., vol. 35, pp. 64-69, Jan. 1997.

[18] A. McAuley, P. Tsuchiya, and D. Wilson, "Fast multilevel heirarchical routing table using content-addressable memory," U.S. Patent 034 444, 1995.

[19] G. Parulkar, D. Schmidt, and J. Turner, "IP/ATM: A strategy for integrating IP with ATM," presented at the SIGCOMM'95, Cambridge, MA.

[20] G. Chandranmenon and G. Varghese, "Trading packet headers for packet processing," IFFE/ACM Trans. Networking, vol. 4, pp. 141-152, Apr. 1996.
[21] Y. Rekhter, B. Davie, D. Katz, E. Rosen, G. Swallow, and D. Farinacci, Tag Switching Architecture Overview Internet Draft, 1996.

[22] M. Degermark, A. Brodnik, S. Carlsson, and S. Pink, "Small forwarding tables for fast routing lookups," presented at the SIGCOMM'97, Cannes, France.

[23] M. Waldvogel, G. Varghese, J. Turner, and B. Plattner, "Scalable high speed IP routing lookups," in Proc. SIGCOMM'97, Cannes, France.

[24] S. Nilsson and G. Karlsson, "Fast address look-up for Internet routers," in Proc. IEEE Broadband Communications'98, Stuttgart, Germany.

[25] V. Srinivasan and G. Varghese, "Fast IP lookups using controlled prefix expansion," ACM TOCS, vol. 17, pp. 1-40, Feb. 1999.

[26] P. Gupta, S. Lin, and N. McKeown, "Routing lookups in hardware at memory access speeds," presented at the IEEE INFOCOM'98, San Francisco, CA.

[27] Micron Technology Inc., Micron, Boise, ID. [Online]. Available HTTP: http://www.micron.com/

[28] Rdram, Rambus, Mountain View, CA. [Online]. Available HTTP: http://www.rambus.com/

[29] J. Hennessey and D. Patterson, Computer Architecture a Quantitative Approach, 2nd ed. San Francisco, CA: Morgan Kaufmann, 1996.

[30] Intel Architecture Software Developer's Manual, Intel, Santa Clara, CA, 1998.

Butler Lampson is an Architect with Microsoft Corporation, Seattle, WA, and an Adjunct Professor at MIT, Cambridge, MA. He has worked on computer architecture, local area networks, raster printers, page description languages, operating systems, remote procedure call, programming languages and their semantics, programming in the large, fault-tolerant computing, transaction processing, computer security, and WYSIWYG editors. He was one of the designers of the SDS 940 time-sharing system, the Alto personal distributed computing system, the Xerox 9700 laser printer, two-phase commit protocols, the Autonet LAN, and several programming languages.

Dr. Lampson received the ACM Software Systems Award in 1984 for his work on the Alto, the IEEE.Computer Pioneer Award in 1996, and the Turing Award in 1992.

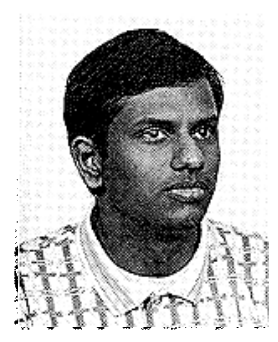

Venkatachary Srinivasan received the B.S. degree in computer science from the Indian Institute of Technology, Madras, India, and is currently working toward the Ph.D. degree at Washington University, St. Louis, MO.

His research involves algorithms to improve Internet-router performance.

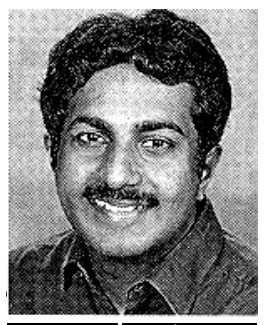

George Varghese (S'95-A'97) received the Ph.D. degree in computer science from MIT, Cambridge, MA, in 1992.

From 1983 to 1993 , he was with the DECNET Architecture and Advanced Development Group, Littleton, MA, where he worked on digital design of network protocols and systems research, in addition to designing algorithms for DEC.NET and GIGAswitch products. He is currently an Associate Professor of Computer Science at Washington University, St. Louis, MO, where he works on distributed algorithms and efficient algorithms for network implementations. His work on network implementations has produced inventions such as timing wheels, fast address lookup algorithms, and scheduling algorithms. He has been awarded ten patents, together with colleagues at DEC, and has applied for five more.

Dr. Varghese was jointly awarded the Sprowls Prize for best thesis in Computer Science at MIT for his Ph.D. dissertation on self-stabilization. He was among two computer scientists to receive the ONR Young Investigator Award in 1996. 